\title{
Counting RSA-Integers
}

\author{
Andreas Decker and Pieter Moree
}

\begin{abstract}
In the RSA cryptosystem integers of the form $n=p \cdot q$ with $p$ and $q$ primes of comparable size ('RSA-integers') play an important role. It is a folklore result of cryptographers that $C_{r}(x)$, the number of integers $n \leq x$ that are of the form $n=p q$ with $p$ and $q$ primes such that $p<q<r p$, is for fixed $r>1$ asymptotically equal to $c_{r} x \log ^{-2} x$ for some constant $c_{r}>0$. Here we prove this and show that $c_{r}=2 \log r$.
\end{abstract}

Mathematics Subject Classification (2000). Primary 11N05; $94 \mathrm{~A} 60$.

Keywords. RSA-integers, primes.

\section{Introduction}

Let $C_{r}(x)$ denote the number of integers $n \leq x$ that are of the form $n=p q$ with $p$ and $q$ primes such that $p<q<r p$, where $r>1$ is an arbitrary real number. In this note we establish the following result.

Theorem 1. As $x$ tends to infinity, we have

$$
C_{r}(x)=\frac{2 x \log r}{\log ^{2} x}+O\left(\frac{r \log (e r) x}{\log ^{3} x}\right) .
$$

Corollary 1. If $r=o(\log x)$, then $C_{r}(x) \sim \frac{2 x \log r}{\log ^{2} x}$, as $x$ tends to infinity.

Since $C_{r}(x) \leq x$, the result is only non-trivial if $r=o\left(\log ^{3} x / \log \log x\right)$.

It is informative to compare Theorem 1 with a classical one due to Landau $[2$, 205-213], who in 1909 proved that $\pi_{2}(x)$, the number of integers $n \leq x$ of the form $n=p q$ with $p$ and $q$ distinct primes satisfies, as $x$ tends to infinity,

$$
\pi_{2}(x) \sim \frac{x \log \log x}{\log x} .
$$

Since then various authors considered the related problem where $n$ consists of precisely $k$ primes factors and $k$ is allowed to vary to some extent with $x$. For a nice survey, see Hildebrand [1]. Note that $C_{x}(x)=\pi_{2}(x)$. 


\section{Proof of Theorem 1}

Let $\pi(x)$ denote the number of primes not exceeding $x$. All we need regarding $\pi(x)$ is the estimate

$$
\pi(x)=\int_{2}^{x} \frac{d t}{\log t}+O\left(\frac{x}{\log ^{3} x}\right)=\frac{x}{\log x}+\frac{x}{\log ^{2} x}+O\left(\frac{x}{\log ^{3} x}\right) .
$$

The integral in this estimate is usually denoted by $\operatorname{Li}(x)$, the logarithmic integral. Using this estimate one easily infers the following stronger form of the so called second theorem of Mertens, which one often encounters in the literature with error term $O\left(\log ^{-1} z\right)$, see, e.g., Tenenbaum [3, p. 16]. For a version of this result with still better error term, see, e.g., Landau [2, p. 201].

Lemma 1. We have

$$
\sum_{p \leq z} \frac{1}{p}=\log \log z+c_{1}+O\left(\frac{1}{\log ^{2} z}\right),
$$

where $c_{1}$ is a constant.

Proof. Write $\pi(z)=\operatorname{Li}(z)+E(z)$. By (1) we have $E(z)=O\left(z \log ^{-3} z\right)$. By Stieltjes integration we find

$$
\sum_{p \leq z} \frac{1}{p}=\int_{2}^{z} \frac{d \pi(t)}{t}=\int_{2}^{z} \frac{d t}{t \log t}+\int_{2}^{z} \frac{d E(t)}{t}=\log \log z+c_{2}+\int_{2}^{z} \frac{d E(t)}{t} .
$$

On noting that

$$
\int_{2}^{z} \frac{d E(t)}{t}=c_{3}+\frac{E(z)}{z}-\int_{z}^{\infty} \frac{E(t) d t}{t^{2}}
$$

and that

the result follows.

$$
O\left(\int_{z}^{\infty} \frac{E(t) d t}{t^{2}}\right)=O\left(\int_{z}^{\infty} \frac{d t}{t \log ^{3} t}\right)=O\left(\frac{1}{\log ^{2} z}\right)
$$

For any prime $p$ we define $f_{p}(x)$ to be the number of primes $q$ such that $p q \leq x$ and $p<q \leq r p$. We clearly have

$$
C_{r}(x)=\sum_{p \leq x} f_{p}(x) .
$$

Lemma 2. We have

$$
f_{p}(x)= \begin{cases}\pi(r p)-\pi(p) & \text { if } p \leq \sqrt{\frac{x}{r}} \\ \pi\left(\frac{x}{p}\right)-\pi(p) & \text { if } \sqrt{\frac{x}{r}}<p \leq \sqrt{x} \\ 0 & \text { if } p>\sqrt{x}\end{cases}
$$

Proof. Since $p<q$ and $p q \leq x$ we infer that $f_{p}(x)=0$ for $p>\sqrt{x}$. So let us assume that $p \leq \sqrt{x}$. Note that we have $p q \leq x$ and $p<q \leq r p$ iff

$$
p<q \leq \min \left\{r p, \frac{x}{p}\right\} \text {. }
$$


We infer that

$$
f_{p}(x)=\pi\left(\min \left\{r p, \frac{x}{p}\right\}\right)-\pi(p)
$$

On noting that

$$
\min \left\{r p, \frac{x}{p}\right\}= \begin{cases}r p & \text { if } p \leq \sqrt{\frac{x}{r}} \\ \frac{x}{p} & \text { if } p>\sqrt{\frac{x}{r}}\end{cases}
$$

the proof is completed.

On combining (2) and Lemma 2 we find that

$$
C_{r}(x)=-\sum_{p \leq \sqrt{x}} \pi(p)+\sum_{p \leq \sqrt{\frac{x}{r}}} \pi(r p)+\sum_{\sqrt{\frac{x}{r}}<p \leq \sqrt{x}} \pi\left(\frac{x}{p}\right) .
$$

The first two sums in the latter expression can be estimated using Lemma 3, the third sum using Lemma 4.

Lemma 3. Let $r \geq 1$. We have

$$
\sum_{p \leq z} \pi(r p)=\frac{r z^{2}}{2 \log ^{2} z}+O\left(\frac{r \log (e r) z^{2}}{\log ^{3} z}\right) .
$$

Proof. First let us assume that $r \leq z^{1 / 4}$. Comparison of $\pi(r p)$ and $\pi(p)$ yields

$$
\pi(r p)=\frac{r p}{\log p}+O\left(\frac{r \log (e r) p}{\log ^{2} p}\right)=r \pi(p)+O\left(\frac{r \log (e r) p}{\log ^{2} p}\right) .
$$

Since $\sum_{p \leq z} p \leq \pi(z) z$ we obtain

$$
O\left(\sum_{p \leq z} \frac{p}{\log ^{2} p}\right)=O\left(\sum_{p \leq z^{1 / 3}} p\right)+O\left(\frac{1}{\log ^{2} z} \sum_{z^{1 / 3}<p \leq z} p\right)=O\left(\frac{z^{2}}{\log ^{3} z}\right) .
$$

From (4) and (5), we infer that

$$
\sum_{p \leq z} \pi(r p)=r \sum_{p \leq z} \pi(p)+O\left(\frac{r \log (e r) z^{2}}{\log ^{3} z}\right)
$$

We see that

$$
\sum_{p \leq z} \pi(p)=\sum_{i \leq \pi(z)} i=\frac{1}{2} \pi(z)(\pi(z)+1)
$$

on noting that as $p$ runs over all primes $\leq z, \pi(p)$ runs over $1,2, \ldots, \pi(z)$. Hence

$$
\begin{aligned}
\sum_{p \leq z} \pi(p) & =\frac{1}{2}\left(\frac{z}{\log z}+O\left(\frac{z}{\log ^{2} z}\right)\right)\left(\frac{z}{\log z}+O\left(\frac{z}{\log ^{2} z}\right)+1\right) \\
& =\frac{z^{2}}{2 \log ^{2} z}+O\left(\frac{z^{2}}{\log ^{3} z}\right),
\end{aligned}
$$

which in combination with (6) yields the result in case $r \leq z^{1 / 4}$. 
In case $r>z^{1 / 4}$ it is enough to show that $\sum_{p<z} \pi(r p)=O\left(r z^{2} \log ^{-2} z\right)$. On noting that $\pi(r p)=O(r p / \log p)$, this estimate easily follows (analogous to the derivation of (5)).

Lemma 4. Suppose that $1 \leq r \leq \sqrt{x}$. Then

$$
\sum_{\sqrt{\frac{x}{r}}<p \leq \sqrt{x}} \pi\left(\frac{x}{p}\right)=\frac{2 x \log r}{\log ^{2} x}+O\left(\frac{x \log ^{2}(e r)}{\log ^{3} x}\right) .
$$

Proof. On writing $p=\sqrt{\frac{x}{a}}$, we find that, for $\sqrt{\frac{x}{r}}<p \leq \sqrt{x}$, we have

$$
\pi\left(\frac{x}{p}\right)=\frac{2 x}{p \log x}+O\left(\frac{x \log (e r)}{p \log ^{2} x}\right) .
$$

We infer from Lemma 1 that

$$
\begin{aligned}
H(x):=\sum_{\sqrt{\frac{x}{r}<p \leq \sqrt{x}}} \frac{1}{p} & =-\log \left(1-\frac{\log r}{\log x}\right)+O\left(\frac{1}{\log ^{2}(x / r)}\right) \\
& =\frac{\log r}{\log x}+O\left(\frac{\log ^{2}(e r)}{\log ^{2} x}\right) .
\end{aligned}
$$

From (7) we infer that

$$
\sum_{\sqrt{\frac{x}{r}<p \leq \sqrt{x}}} \pi\left(\frac{x}{p}\right)=2 H(x) \frac{x}{\log x}+O\left(H(x) \frac{x \log (e r)}{\log ^{2} x}\right) .
$$

This, together with the estimate for $H(x)$ we determined, yields the result.

We end with the proof of Theorem 1.

Proof. For $r>\sqrt{x}$ we have $C_{r}(x) \leq x=O\left(r \log (e r) x \log ^{-3} x\right)$ and so Theorem 1 is trivially true for this $r$-range. Now assume that $r<\sqrt{x}$. By Lemma 3 (with $r=1$ and $z=\sqrt{x}$ ) we have

$$
\sum_{p \leq \sqrt{x}} \pi(p)=\frac{2 x}{\log ^{2} x}+O\left(\frac{x}{\log ^{3} x}\right) .
$$

Lemma 3 with $z=\sqrt{\frac{x}{r}}$ yields

$$
\sum_{p \leq \sqrt{\frac{x}{r}}} \pi(r p)=\frac{2 x}{\log ^{2} x}+O\left(\frac{x r \log (e r)}{\log ^{3} x}\right) .
$$

The proof now follows on inserting the latter two estimates and the estimate given in Lemma 4 in the equality (3). 


\section{Acknowledgements}

(A. Decker:) This note was written whilst the first author did an internship at the Max-Planck-Institut für Mathematik in Bonn. The other interns Alexander Bridi, Patrizia Dressler, Silke Glas and Thorge Jensen also contributed to this paper with different suggestions for improvement and some own calculations. Last, but not least, the results on this problem are also due to the good understanding of the interns, their good relationship with their coordinator, the second author, and the pleasant atmosphere at the MPIM in Bonn.

(P. Moree:) The problem of estimating RSA-integers was proposed by Benne de Weger (TU Eindhoven) to the second author, who gave it as one of several problems to the above interns. It was in essence solved by Andreas Decker. The proof presented here is shorter and somewhat different and has absolute implicit errors instead of $r$-dependent ones.

\section{References}

[1] A. Hildebrand, On the number of prime factors of an integer, Ramanujan revisited (Urbana-Champaign, Ill., 1987), 167-185, Academic Press, Boston, MA, 1988.

[2] E. Landau, Handbuch der Lehre von der Verteilung der Primzahlen, Chelsea Publishing Co., New York, 1953.

[3] G. Tenenbaum, Introduction to analytic and probabilistic number theory, Cambridge Studies in Advanced Mathematics 46, Cambridge University Press, Cambridge, 1995.

Andreas Decker

Achtern Diek 32

D-49377 Vechta

Germany

e-mail: andreasd@uni-bonn.de

Pieter Moree

Max-Planck-Institut für Mathematik

Vivatsgasse 7

D-53111 Bonn

Germany

e-mail: moree@mpim-bonn.mpg.de

Received: December 18, 2007.

Revised: January 8, 2008. 